\title{
Executive function and self-regulated exergaming adherence among older adults
}

\section{Cay Anderson-Hanley ${ }^{*}$, Paul J. Arciero' ${ }^{2}$, Nicole Barcelos ${ }^{1}$, Joseph Nimon ${ }^{1}$, Tracey Rocha ${ }^{3}$, Marisa Thurin ${ }^{1}$ and Molly Maloney ${ }^{1}$}

\author{
${ }^{1}$ Healthy Aging and Neuropsychology Lab, Department of Psychology, Union College, Schenectady, NY, USA \\ 2 Human Nutrition and Metabolism Laboratory, Health and Exercise Sciences Department, Skidmore College, Saratoga Springs, NY, USA \\ ${ }^{3}$ The Division of Counseling Psychology, Department of Educational and Counseling Psychology, School of Education, University at Albany, Albany, NY, USA
}

Edited by:

Sean P. Mullen, University of Illinois at Urbana-Champaign, USA

Reviewed by:

John Best, University of British

Columbia, Canada

Ann Elisabeth Vandenberg, Emory

University, USA

${ }^{*}$ Correspondence:

Cay Anderson-Hanley, Healthy

Aging and Neuropsychology Lab,

Department of Psychology, Union

College, 807 Union Street,

Schenectady, 12308 NY, USA

e-mail: andersoc@union.edu
The rise in dementia and the evidence of cognitive benefits of exercise for the older adult population together make salient the research into variables affecting cognitive benefit and exercise behavior. One promising avenue for increasing exercise participation has been the introduction of exergaming, a type of exercise that works in combination with virtual reality to enhance both the exercise experience and health outcomes. Past research has revealed that executive function (EF) was related to greater use of self-regulatory strategies, which in turn was related to greater adherence to exercise following an intervention (McAuley et al., 2011). Best et al. (2014) found improvement in EF related to adherence to exercise post- intervention. Anderson-Hanley et al. (2012) found that for older adults aerobic exergaming yielded greater cognitive benefit than traditional exercise alone; however, questions remain as to the possible impact of greater cognitive benefit and other factors on participants' involvement in exercise following the end of an intervention. The current study presents follow-up data exploring the relationship between EF, self-regulation, and exercise behavior in the post-intervention (naturalistic) period. Herein, it was predicted that higher EF at the start of the naturalistic window, would predict subsequent exercise with an exergame. Contrary to expectations, results suggest that those with poorer EF are likely to exergame more frequently. The results of this study contradict previous literature, but suggest an interesting relationship between $\mathrm{EF}$, self-regulation, and exercise behaviors when exergaming is employed, particularly with older adults with some cognitive decline. We hypothesize that other factors may be at work, perhaps expectation of cognitive benefit might act as a unique motivator.

Keywords: exergame, aerobic exercise, cognition, older adults, self-regulation, executive function

\section{INTRODUCTION}

The positive impact of exercise on cognition has been well documented in the scientific literature (Kramer et al., 1999; Colcombe et al., 2004; Hogan et al., 2013). Longitudinal research in Europe indicated that people aged 50 years and older who participated in any kind of physical activity showed less cognitive decline after 2.5 years than those who performed no physical activity (Aichberger et al., 2010). Randomized controlled trials (RCTs) and exercise intervention studies, moreover, have revealed that both aerobic exercise (Colcombe and Kramer, 2003; Smith et al., 2010) and resistance training (Cassilhas et al., 2007; Liu-Ambrose et al., 2010; Nagamatsu et al., 2012) improve cognition, especially aspects of executive function (EF) including planning, decision making, and inhibition. Nonetheless, research reveals that only $4 \%$ of adults exercise at the recommended frequency and intensity (Gordon-Larsen et al., 2004).

Given the well-established evidence-base suggesting that exercise offers cognitive benefit for the older adult population in particular (Colcombe et al., 2004; Hindin and Zelinski, 2012), and the concomitant rise in rates of dementia (Steffens et al., 2009), research efforts must now turn to understanding variables which may predict this populations' participation in and adherence to a fitness regimen. An understanding of the variables that predict involvement in exercise behavior (both during and after prescribed interventions) could lead to more targeted interventions for older adults and, therefore, to more exercise and cognitive benefit.

Various theoretical approaches have evolved that attempt to understand behavioral constructs and related variables that may moderate the relationship between exercise intervention and maintenance of exercise behavior. While our research on the cognitive benefits of exercise for older adults led us to examine factors related to adherence within that context, it is important to note that significant contributions to the work on the role of $\mathrm{EF}$ in exercise adherence has resulted from a broader set of research. That body of literature has pioneered the role of EF in self-regulation and examined that relationship in diverse 
topics ranging from health behaviors and consumer research on decision making, lending further support to the notion that EF plays a critical role in the maintenance of desirable behaviors (e.g., in treatment of substance abuse, in pain management, recovery from brain injury; Solberg Nes et al., 2009; Hofmann et al., 2008; Hunt et al., 2013; Bickel et al., 2014). Generally, EF is the mechanism that allows a person to make choices about behaviors that may not immediately positively affect them, but will have positive effects in the long run (e.g., suppressing an unconsidered impulse to buy something expensive, going to the gym daily to make incremental steps toward health). Executive function requires a host of underlying cognitive, biological, and social processes to function (Hall and Fong, 2007). It is hypothesized that EF may play a major role in various behaviors, but especially self-motivated exercise behaviors, because it is implicated in the delay of gratification and participation in activities which may not yield immediate external reward (Hall and Fong, 2007).

One approach, the so-called social-cognitive approach, is undergirded and perpetuated by findings that variables like selfefficacy and social support (Chogahara et al., 1998; McAuley and Blissmer, 2000) may be mechanisms through which exercise behaviors occur in the absence of other extrinsic motivations (e.g., rewards for participating in employer health and wellness program). A variant of those theories cited in the social and health psychology literature, the Theory of Planned Behavior (TBP), has been suggested to play a role in health-related behaviors (Godin and Kok, 1996). The theory attempts to explain how people's attitudes and beliefs about objects and themselves influence their participation in health-relevant behaviors (Ajzen, 1985). Selfefficacy is a core control belief in the TBP model and has been robustly demonstrated to play a role in people's engagement in exercise behaviors.

Another approach to analyzing maintenance of exercise behavior post-exercise intervention is a more biological approach that has been motivated by existent evidence suggesting that exercise structurally and functionally alters the brain (Colcombe et al., 2006; Hillman et al., 2008). Research from this neurocognitive approach focuses, in particular, on the role of top-down processes, including EF in adherence to exercise behaviors. The temporal self-regulation theory (TST), a theory of the neurocognitive approach, provides a theoretical framework through which exercise intervention, EF, and regimen adherence may relate. Generally, TST offers an explanation for how people decide to engage in immediate behaviors that yield later, but not immediate, effects. According to TST, there are many underlying social, biological, and cognitive processes that work together to enable a person to engage in behaviors that will produce delayed benefits; these processes are crucial for the motivation required to continue a regular exercise regimen (Hall and Fong, 2007).

In a recent RCT and subsequent follow-up study of these phenomena, Best et al. (2014) examined the relationship between EF and adherence to an exercise routine post-fitness intervention. The researchers hypothesized that increased EF (due to an exercise intervention) would predict continued participation in exercise once the intervention ceased. Results revealed that women who had experienced greater EF benefit from the exercise intervention were more likely to adhere to an exercise routine in the year following the cessation of the intervention. These results were unaffected by any potential covariates (i.e., age, education, depression, and MOCA scores). Best et al. (2014) research suggests that functional changes in brain processes, especially EF, may subsequently impact maintenance of exercise behavior.

McAuley et al. (2011) conducted a year-long exercise intervention with a sample of older adults and subsequently analyzed exercise behaviors in the year following the intervention. Expanding on prior research and connecting aspects of the social-cognitive approach with the neurocognitive approach, they included various measures of EF, such as working memory, inhibition, and task switching, while also measuring the use of self-regulatory strategies and self-efficacy. The results revealed that specific types of EF (i.e., task switching and inhibition) were related to greater use of self-regulatory strategies, which in turn was related to higher self-efficacy; this higher self-efficacy predicted greater adherence to an exercise routine in the year following the cessation of the exercise intervention. McAuley et al. (2011) research is especially important for understanding health-related behaviors, because it suggests that both social/personal characteristics and neurocognitive processes play a role in the maintenance of healthful behavior, such that self-efficacy may mediate elements of EF to influence exercise behavior.

The current research aims to expand on research such as Best et al. (2014) and McAuley et al. (2011) by examining how change in EF relates to and plays an important role in exercise adherence. The question investigated in the current research is whether or not EF may be related to people's participation in an exercise behavior; in particular, after the conclusion of a prescriptive exercise intervention period. In our previous research we examined the impact of exergaming ("cybercycling"1) on cognitive functions in older adults (Anderson-Hanley et al., 2012). Exergaming is a type of exercise that works in combination with virtual reality and/or videogames to enhance both the exercise experience and possibly also relevant mental processes (e.g., Wii Fit and X-Box Kinect) and is a promising avenue for increasing exercise participation. We found that exergaming yielded greater cognitive benefit, specifically EF, than riding a stationary bike alone (despite a similar dose of exercise in terms of miles, minutes, and intensity). Crucial questions remain, however, as to the possible impact of such a cognitive benefit and other relevant factors on participants' involvement in exercise behaviors following the end of the exergaming intervention. The current research is a follow-up study which further explores the relationship between exercise behavior, self-regulation and executive control in the post-intervention period of the above noted cybercycle study.

\section{HYPOTHESIS}

As demonstrated by prior research (McAuley et al., 2011; Best et al., 2014), it was predicted that EF at the start of the naturalistic exercise window (thus at the end of the cybercycle randomized

\footnotetext{
${ }^{1}$ stationary bike with a virtual reality screen that displays interactive bike tours with on-screen competition or a videogame.
} 
trial), would predict frequency of exercise on the cybercycle postintervention.

\section{METHODS SAMPLE CHARACTERISTICS}

In a previous randomized clinical trial, 102 older adults were recruited from local independent living facilities and randomized to one of two exercise conditions: physical exercise alone (traditional stationary bike) vs. interactive virtual reality-enhanced stationary cycling (cybercycle). Individuals with known neurological disorders (e.g., Alzheimer's disease) or significant functional disabilities that would restrict participation in physical exercise or cognitive testing were excluded from participation; written physician approval to exercise was required. The study was approved by a human subjects review committee and written informed consent was obtained for all enrollees; the study was registered as a national clinical trial ${ }^{2}$ (NCT01167400). Sixty-three participants completed the 3-month randomized portion of the study (results reported elsewhere; see Anderson-Hanley et al., 2012), and 51 were willing and able to continue exercising during the subsequent naturalistic exercise period and reported for final testing after that 6-month window. The average age of the group that participated during the naturalistic window $(n=51)$ was 79.0 years $(\mathrm{SD}=8.4)$ and their average years of education was 13.7 $(\mathrm{SD}=2.5) ; 71 \%$ were women and the sample was predominantly Caucasian $(>90 \%)$. Data from 30 participants were in the present analyses; they include those who had also completed the RCT, completed evaluations at the end of the naturalistic exercise window, had data on the variables of interest herein, and had valid exercise data (those with zero ride frequency who had a physical injury or had moved away were dropped from the analyses). Outliers were also examined and consistent with Best et al. (2014), results are also presented with one outlier removed.

\section{PROCEDURES}

During the RCT, participants were randomly assigned to either the traditional stationary bike group or the cybercycle group. Participants in both groups rode the same recumbent stationary bike, but in the cybercycle condition a virtual reality screen was active and participants interacted with a virtual bike tour, steering and competing with avatars. All participants were encouraged to gradually increase their total time of exercise to $45 \mathrm{~min}, 5$ days a week in their respective condition, consistent with American College of Sport Medicine (ACSM) standards (Garber et al., 2011). Participants were instructed to adhere to their assigned exercise condition for 3 months. After each exercise session participants documented their ride behaviors on a paper log. Ride logs were collected from the study sites and used to calculate ride statistics (frequency, intensity, duration). The average number of rides for the cybercycle group and control groups were $51.3(\mathrm{SD}=3.32)$ and $53.3(\mathrm{SD}=3.14)$, with average durations of $35.5 \mathrm{~min}$ and $33.8 \mathrm{~min}$, respectively. After 3 months in their randomized conditions, participants were invited to engage in naturalistic exercise for 6 months.

${ }^{2}$ clinicaltrials.gov
During the naturalistic exercise window, participants in both the control and cybercycle conditions were encouraged to exercise 5 days a week for 45 min per session while using the cybercycle in its fullest capacity (e.g., navigating various bike tours or playing a dragon chase videogame). It is the naturalistic exercise window that is the focus of this report. Again, paper ride logs were collected from the study sites and used to calculate ride statistics as noted above. We chose to focus on the middle 3 months of the 6-month ride window due to missing data from some sites at the beginning and end of the naturalistic window. Using a 3 -month window also provided a comparable exercise window to that studied in the RCT.

\section{MEASURES}

Tests of cognitive functioning were administered at enrollment (baseline), 1 month after that (pre-intervention), and then again after the 3-month randomized exercise conditions (post-intervention/pre-naturalistic exercise window). The current research examines the relationship between EF postintervention and exercise behavior during the follow-up naturalistic window, during which participants were encouraged to utilize the cybercycle in its full capacity.

\section{Executive function}

The original study utilized a full battery of neuropsychological tests to characterize the sample and track changes; alternate forms were used at each time point. Consistent with recent data published on this topic (Best et al., 2014), the current analysis focused on the Stroop Test (Stroop, 1935). The Stroop Test has proven to be one of the most effective measures of EF as the brain areas activated during this task are associated with costbenefit decision making and impulse control (Strauss et al., 2006). A shortened, 40-item version of the Stroop was used, presenting colored blocks first (Stroop A), followed by black text color names (Stroop B), and finally color names printed in contrasting colored ink (Stroop C; adapted from Van der Elst et al., 2006). In an effort to partially replicate the recently reported work of Best et al. (2014), Stroop C-Stroop A was used as a measure of EF, and it was the change in this EF variable over the course of the initial RCT that was the focus of our analyses. Given that that time in seconds is the outcome, greater Stroop C-A scores indicate worse EF; thus, a negative change score (post-RCT minus pre-RCT), indicates improving EF.

\section{Exercise attitudes measures}

In addition to cognition, other variables were measured to examine possible influences on exercise behaviors; in particular: selfefficacy, perceived benefits and barriers to physical activity, and attitudes and motivations towards physical activity and the cybercycle specifically. Self-efficacy was measured using the Self Efficacy for Physical Activity Survey (SEPAS; Sallis et al., 1988); questions assessed how confident participants were that they could complete exercise behavior in the face of various barriers such as needing to wake up early, set aside time, and continue adhering to a routine during stressful times. The Exercise Benefits/Barriers Scale (EBBS; Sechrist et al., 1987) was used to measure the degree to which participants saw both benefits and barriers in exercise. Questions 
assessed whether participants thought exercise would benefit variables such as their physical and mental health and whether participants perceived a number of barriers, such as embarrassment, in adhering to an exercise routine. Lower scores indicate greater endorsement. The Social Support and Exercise Survey (SSES; Sallis et al., 1987) was used to measure the degree to which participants received support from family members and friends for exercise endeavors. Participants' motivations for exercising in general and for completing the cybercycle study, specifically, were assessed. The Motives for Physical Activities Measure-Revised (MPAM-R; Ryan et al., 1997) was used to evaluate general exercise motivations. Participants rated the degree to which they were exercising for various reasons including wanting to be fit, look better, stay in shape, and because others motivating them.

\section{Motivation to exercise for cognitive benefit}

Given unexpected findings noted below, we probed our dataset for items that tapped participant motivation to exercise for cognitive benefit (MECB). Two items were identified that appeared to capture this construct. One item was taken from the Cybercycling Attitudes Test (CAT), which was developed by Nimon et al. (2009) to measure attitudes towards the cybercycle intervention: "Exercising on the cybercycle will make me think more clearly" (Likert scale 0-5, disagree to agree). The other item (\#34) was taken from the EBBS: "Exercising increases my mental alertness" (Likert scale 1-4, agree to disagree). The EBBS item was reversed scored and scaled to match the CAT. These items were found to be somewhat correlated $(r=0.33)$ and summed to form a two-item measure of MECB.

\section{Other measures}

Participants were characterized as meeting criteria for mild cognitive impairment (MCI), if they scored $-1.5 \mathrm{SD}$ on three domains in the RCT's comprehensive neuropsychological battery (Jak et al., 2009). Participants' health was assessed using the Pennebaker Inventory of Limbic Languidness (the PILL) to assess participants' experience of common health symptoms (PILL; Pennebaker, 1982). The PILL assesses how often people have recently experienced various common symptoms and sensations, such as swollen joints, asthma, and dizziness.

\section{STATISTICAL ANALYSES}

Partial correlations were conducted in SPSS version 19 (IBM Corporation, 2012). Descriptive statistics were computed in EXCEL (Microsoft Corporation, 2010).

\section{RESULTS}

\section{PRELIMINARY RESULTS}

The current sample $(n=30)$ was compared with those who completed the original RCT but did not participate in the naturalistic window $(n=33)$. No significant differences were found on key variables (i.e., age, education, MCI status, EF) between the sample herein of those continuing to be followed in the study after the RCT and those from the original sample who did not continue on to participate in the naturalistic exercise window. Descriptive data is presented in Table 1.

\section{PREDICTORS OF EXERCISE ADHERENCE}

Partial correlations (list-wise) were conducted to examine the relationship between exercise frequency (number of rides on the cybercycle based on participants ride logs; average duration $=35.7 \mathrm{~min}$ and average intensity $=130 \mathrm{kcal}$ ) and change in EF (formula above adapted from Best et al., 2014), as well as exercise attitudes (benefits, barriers, social support, self-efficacy, and motivations), other possible predictors (MCI status, physical illness), all while controlling for randomized group assignment, age, education, and baseline EF.

Initial analyses including all eligible cases $(n=30)$ revealed a trend-level negative correlation between EF improvement (during prior RCT) and subsequent exercise frequency; indicating, that contrary to expectations, those with declining EF at the start of the naturalistic window went on to exercise more frequently than those who had made EF gains $(r=0.38 ; p=0.0549)$. As noted above, consistent with Best et al. (2014) approach of excluding outliers, one extreme outlier was identified and removed from analyses. The reanalysis confirmed a strong inverse relationship between prior EF decline and later exercise adherence $(r=0.43$; $p=0.03)$. Partial correlation results are reported in Table 2 .

Because of the above noted unexpected inverse relationship between EF improvement and exercise adherence, we considered the possibility that our sample might differ from typical exercise studies in that the intervention was novel (combining interactive mental and physical exercise), and participants had received information regarding the possible cognitive benefits of exercise. Furthermore, since our sample was older than those in the study by Best et al. (2014) and many other typical exercise RCTs, we did have a greater proportion that was already starting to experience some cognitive decline and might be thus more motivated to try to improve their function and slow decline. We wanted to evaluate the possible impact of participants' motivation to exercise due to this unique feature of the RCT intervention and reviewed our data set for specific items that tapped participant motivation to exercise for possible cognitive benefits (see items identified and selected under Section Measures above). Motivation to exercise for cognitive benefit was thus also evaluated for its relationship to outcomes, and while statistically non-significant, indicates a possible trend $(r=0.35 ; p=0.08)$.

A significant relationship between self-efficacy for physical activity and ride frequency $(r=0.52 ; p=0.01)$ was also seen, in that individuals with higher exercise self-efficacy were found to exercise more frequently during the naturalistic window compared to those with lower personal efficacy for physical activity. This finding is in line with TPB and supports previous findings (Ajzen, 1985; McAuley and Blissmer, 2000; McAuley et al., 2011). Additionally, a significant inverse relationship was found between motivation to exercise for physical appearance and exercise adherence $(r=-0.41, p=0.04)$.

\section{DISCUSSION}

Results of a small sample of older adults exercising following a 3-month randomized trial of exergaming vs. traditional exercise, suggests a somewhat curious and unexpected relationship between $\mathrm{EF}$ and self-regulated engagement in exergaming. The relationship found herein suggests 


\begin{tabular}{|c|c|c|c|c|c|c|c|}
\hline & \multicolumn{3}{|c|}{ Current sample ${ }^{a}$} & \multicolumn{4}{|c|}{ Non-continuing sample ${ }^{b}$} \\
\hline & ave & SD & $n$ & ave & SD & $n$ & $p$ \\
\hline \multicolumn{8}{|l|}{ Demographics } \\
\hline group (\% cybercycle group) & $60 \%$ & & 30 & $36 \%$ & & 33 & 0.06 \\
\hline age & 79.5 & 9.2 & 30 & 78.5 & 8.5 & 33 & 0.64 \\
\hline education (yrs) & 14.1 & 3.2 & 30 & 13.6 & 1.9 & 33 & 0.45 \\
\hline sex (\% female) & $67 \%$ & & 30 & $79 \%$ & & 33 & 0.29 \\
\hline $\mathrm{MCl}$ classification & $40 \%$ & & 30 & $21 \%$ & & 33 & 0.11 \\
\hline \multicolumn{8}{|l|}{ Exercise outcome } \\
\hline ride frequency & 21.0 & 20.2 & 30 & 27.8 & 25.8 & 23 & 0.29 \\
\hline \multicolumn{8}{|l|}{ Executive function } \\
\hline Stroop C-A & 29.24 & 14.6 & 30 & 29.71 & 23.7 & 31 & 0.93 \\
\hline Stroop C-A (prior intervention change: post-pre) ${ }^{\mathrm{C}}$ & -3.99 & 0.32 & 30 & 0.32 & 12.02 & 29 & 0.17 \\
\hline \multicolumn{8}{|l|}{ Exercise attitudes } \\
\hline Exercise benefits and barriers scale (benefits) & 61.9 & 7.2 & 30 & 59.7 & 13.6 & 26 & 0.43 \\
\hline Exercise benefits and barriers scale (barriers) & 35.7 & 7.6 & 30 & 39.54 & 8.5 & 26 & 0.08 \\
\hline Social support for exercise scale (family) & 22.8 & 12.6 & 30 & 23.1 & 10.2 & 25 & 0.93 \\
\hline Social support for exercise scale (friends) & 24.6 & 11.3 & 30 & 23.9 & 11.1 & 22 & 0.83 \\
\hline Self-efficacy physical activity scale (total) & 46.3 & 9.4 & 30 & 46.5 & 10.3 & 31 & 0.94 \\
\hline Motivation for physical activity measure (enjoy) & 35.3 & 9.3 & 30 & 33.1 & 12.0 & 30 & 0.43 \\
\hline Motivation for physical activity measure (appearance) & 29.4 & 7.7 & 30 & 27.8 & 9.8 & 30 & 0.48 \\
\hline Motivation for physical activity measure (fitness) & 32.0 & 4.2 & 30 & 30.2 & 5.8 & 30 & 0.17 \\
\hline Motivation to exercise for cognitive benefit & 9.0 & 1.4 & 30 & 8.1 & 2.9 & 32 & 0.14 \\
\hline
\end{tabular}

Note: Data on measured variables were collected post-randomized exercise/pre-naturalistic exercise.

a The "Current Sample" refers to those who completed the original RCT and the follow-up (even if they had zero rides).

${ }^{b}$ The "Non-continuing Sample" refers to those who completed the original RCT, but not the follow-up.

cUsed change in difference score formula consistent with Best et al. (2014).

an inverse relationship between EF improvement and the frequency of exergaming, which contradicts previous literature on the effects of EF on exercise adherence via increased self-regulation (McAuley et al., 2011; Best et al., 2014). Among this particular sample of older adults, decline in EF during a prior exercise intervention was associated with greater exercise frequency during a followup window.

One hypothesis to explain this apparent inverse relationship is that given the nature of this sample (older than some exercise trial samples) and the specific type of exercise involved (i.e., exergaming, involving interactive mental and physical exercise), the exergaming may have been more motivating for participants who were already experiencing some cognitive decline as they may have been hopeful it would yield cognitive benefit. This would fit with TST, as participants experiencing some cognitive decline might be more willing to engage immediate exercise given the possibility that they might yield cognitive and physical health benefits eventually. However, our examination of items related to MECB did not yield a statistically significant relationship, but a possible trend in the expected direction with the outcome of exercise behavior (cybercycle rides). It may be that our post hoc generated measure of motivation was not sufficient to capture this factor or that of other factors may be more important in determining the relationship between EF and exercise behavior. Physical appearance was the only motivation item that produced a significant relationship with exercise behavior, in that motivation to exercise for physical appearance was inversely related to exercise adherence. Withall et al. (2011) found a similar finding in their examination of individuals' motivations for engaging in an organized physical activity. One hypothesis for their finding is that those who indicated they were motivated to exercise for physical appearance may have dropped out if they did not see immediate improvements in their appearance. Additionally, previous studies which have found motivation to exercise for physical appearance to decrease with age (Trujillo et al., 2004; Dacey et al., 2008), suggesting that our population is likely more motivated by other factors. In keeping with TST, it may be that participants were more motivated to exercise due to intrinsic factors, rather than extrinsic reasons. Future research is needed to examine the specific relationship between EF, self-regulation, and exercise behaviors when exergaming is employed. It may be important to develop and measure motivation for exercise to benefit cognition, and to specifically focus on the relationship among these variables when targeting exercise interventions for older adults with some cognitive impairment.

Another possible explanation for the unexpected inverse relationship between EF and exercise behavior is that those who did not exercise on the cybercycle during the naturalistic exercise period may have engaged in other physical activities, such as walking on a treadmill. This was not prospectively assessed and is a limitation of this study; rather we depended on participants to sustain the expectation of "no changes to current physical activity" as stated at the beginning of the study. However, a few 
Table 2 | Partial correlations with ride frequency

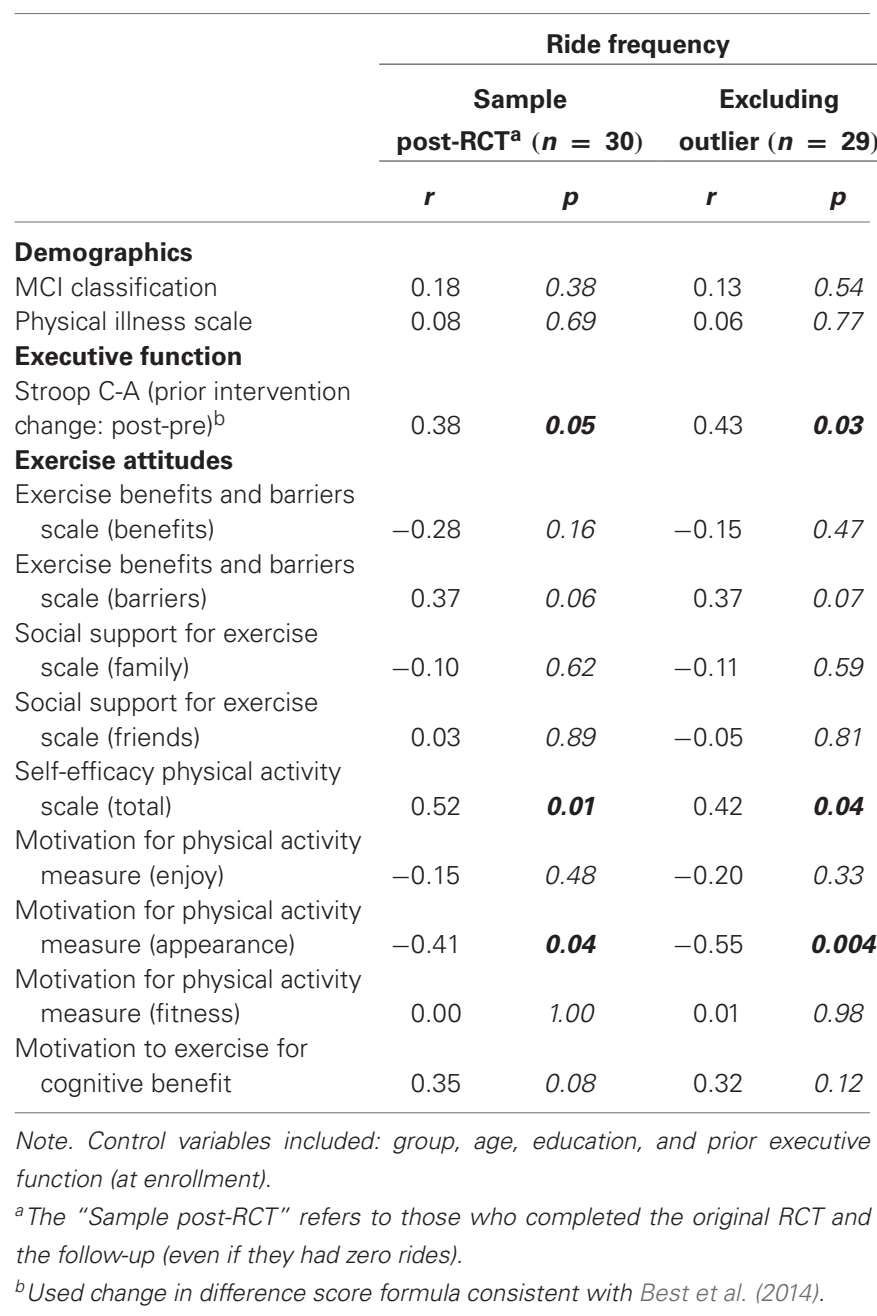

participants noted in the bike logs that they had participated in other physical activity (e.g., treadmill time). Future research should prospectively and specifically track other physical activity.

Another consideration in interpreting these findings is that family members and caregivers of older adults experiencing some cognitive decline may serve as external motivators, changing selfregulation into other-regulation. For example, a spouse may be concerned that their loved one is not as mentally sharp and fears further decline into dementia, so they may increase their own efforts to facilitate a participant showing up for their allotted exercise slot on the cybercycle. Although we did assess social support for exercise, it is unclear whether some participants would have been able to report this in a way that would capture the possibly subtle, but substantial impact of instrumental assistance some caregivers may have been providing. Future research might find a way to clarify this possible impact on regulation by extension.

Furthermore, it is possible that these results do not entirely contradict the recent report of Best et al. (2014), but rather may illuminate a more complex relationship between changing cognition across later life and exercise behaviors. The sample in their study was comprised of women nearly 70 years old with normative performance on a screen for MCI, whereas our sample was on average a decade older and more impaired overall ( $40 \%$ met criteria for MCI). Thus, some in our sample would have been slipping cognitively regardless of intervention, and while exercise may not have "improved" $\mathrm{EF}$ in an absolute way for some, it may have slowed decline (e.g., Lautenshlager et al., 2008). Our sample may have overlapped with Best's sample, including some normative participants for whom a positive linear relationship with exercise adherence could apply; however, result may be clouded by the mix of more impaired participants for whom may exist a different relationship between EF and adherence. A larger sample, perhaps also a matching sample of normative and MCI participants, would be needed to discern the possible merits or lack thereof of this speculation.

\section{STRENGTHS}

These participants engaged previously in two randomized exercise conditions that provided an interesting comparison since they both involved the same aerobic exercise, but varied mental interactivity. Other studies may assign participants to groups that involve different forms of exercise that may not be comparable on a key variable such as HR (Best et al., 2014), while others may compare an older adult group that is engaging in exercise and a group that is engaging in a non-physical activity (Evers et al., 2011). Participants in the present research engaged in the same type of physical activity during the RCT, thus providing an equivalent starting point in terms of recent prior physical activity; that is, no group was inadvertently advantaged/disadvantaged by having to "catch up" on aerobic exercise, which could have affected results. Instead, during the naturalistic exercise window studied herein, all participants were free to immerse themselves in a virtual tour while engaging aerobic exercise to which their body would have already become accustomed.

Unlike some previous research, there was no transportation barrier for these participants. The equipment and all necessary materials were available in the participant's residences for ease of access. This greatly reduced the amount of effort required of participants which has been found to deter exercising in previous studies, such as bad weather or having to take public transportation (Evers et al., 2011).

\section{LIMITATIONS}

A major limitation of this study was the impact of randomized experience in the control (bike only) and experimental group (cybercycle). By giving the participants the freedom to choose which way they would like to use the bike after the initial 3-month intervention period, those participants that were on the stationary bike seem to have engaged in the cybercycle more frequently, perhaps in part due to its novelty. Those who had been originally assigned to the cybercycle may have lost interest after having been required to exercise on it for 3 months, thus either discontinuing or perhaps choosing different (unspecified/non-bike) forms of exercise. As noted above, this limitation should be addressed in future research by prospective assessment of other forms of physical activity. Other limitations include the small sample size and the variability of the sample (some clearly normative older 
adults, with some starting to experience cognitive decline). The use of multiple statistical tests also may have led to escalating Type I error rates.

\section{FUTURE RESEARCH}

Future research might be able to further explore these preliminary findings by examining a larger sample of older adults with some cognitive decline present, and carefully measuring EF, self- and other-regulation, exercise behaviors (as targeted, but also with consideration of non-compliance), and if examining a novel form of exercise, such as exergaming, consider measuring specific variables that could be salient (such as MECB). A larger sample would allow more sophisticated analysis of mediation and moderator models, as well as consideration of change over time that was precluded here by a small sample.

\section{CONCLUSION}

This report presents preliminary evidence of an unexpected inverse relationship between $\mathrm{EF}$ and exercise adherence among older adults. It was hypothesized that that this relationship might be explained by some cognitive decline present in the sample and increased motivation to exercise to protect cognitive function. The latter being an outcome perceived to be possible via the unique interactive mental and physical exercise offered in the exergame (virtual reality stationary bike) utilized herein. Further research is warranted with a larger sample, enabling comparison of possible subgroups (e.g., normative vs. MCI), and examining additional factors affecting motivation and adherence (e.g., exercising for cognitive benefit and/or the possible instrumental role of caregivers in adherence).

\section{REFERENCES}

Aichberger, M. C., Busch, M. A., Reischies, F. M., Stro'hle, A., Heinz, A., and Rapp, M. A. (2010). Effect of physical inactivity on cognitive performance after 2.5 years of follow-up: longitudinal results from the survey of health, ageing and retirement (SHARE). GeroPsych 23, 7-15. doi: 10.1024/1662-9647/a000003

Ajzen, I. (1985). "From intentions to actions: a theory of planned behavior," in Action Control: From Cognition to Behavior, eds J. Kuhl and J. Beckmann (Berlin Heidelberg: Springer), 11-39.

Anderson-Hanley, C., Arciero, P. J., Brickman, A. M., Nimon, J. P., Okuma, N., Westen, S. C., et al. (2012). Exergaming and older adult cognition: a cluster randomized clinical trial. Am. J. Prev. Med. 42, 109-119. doi: 10.1016/j.amepre. 2011.10.016

Best, J. R., Nagamatsu, L. S., and Liu-Ambrose, T. (2014). Improvements to executive function during exercise training predict maintenance of physical activity over the following year. Front. Hum. Neurosci. 8:353. doi: 10.3389/fnhum.2014. 00353

Bickel, W. K., Johnson, M. W., Koffarnus, M. N., MacKillop, J., and Murphy, J. G. (2014). The behavioral economics of substance use disorders: reinforcement pathologies and their repair. Annu. Rev. Clin. Psychol. 10, 641-677. doi: 10. 1146/annurev-clinpsy-032813-153724

Cassilhas, R. C., Viana, V. A. R., Grassmannetal, V., Santos, R. T., Santos, R. F., Tufik, S., et al. (2007). The impact of resistance exercise on the cognitive function of the elderly. Med. Sci. Sports Exerc. 39, 1401-1407. doi: 10.1249/mss. 0b013e318060111f

Chogahara, M., Cousins, S., and Wankel, L. (1998). Social influences on physical activity in older adults: a review. J. Aging Phys. Act. 6, 1-17.

Colcombe, S. J., Erickson, K. I., Scalf, P. E., Kim, J. S., Prakash, R., McAuley, E., et al. (2006). Aerobic exercise training increases brain volume in aging humans. J. Gerontol. A Biol. Sci. Med. Sci. 61, 1166-1170. doi: 10.1093/gerona/61.11.1166

Colcombe, S., and Kramer, A. F. (2003). Fitness effects on the cognitive function of older adults: a meta-analytic study. Psychol. Sci. 14, 125-130. doi: 10.1111/14679280.t01-1-01430
Colcombe, S. J., Kramer, A. F., Erickson, K., Scalf, P., McAuley, E., Cohen, N. J., et al. (2004). Cardiovascular fitness, cortical plasticity and aging. Proc. Natl. Acad. Sci. U S A 101, 3316-3321. doi: 10.1073/pnas.0400266101

Dacey, M., Baltzell, A., and Zaichkowsky, L. (2008). Older adults' intrinsic and extrinsic motivation toward physical activity. Am. J. Health Behav. 32, 570-582. doi: 10.5555/ajhb.2008.32.6.570

Evers, A., Klusmann, V., Schwarzer, R., and Heuser, I. (2011). Improving cognition by adherence to physical or mental exercise: a moderated mediation analysis. Aging Ment. Health 15, 446-455. doi: 10.1080/13607863.2010.543657

Garber, C. E., Blissmer, B., Deschenes, M. R., Franklin, B. A., Lamonte, M. J., Lee, I. M., et al. (2011). American college of sports Medicine position stand. Quantity and quality of exercise for developing and maintaining cardiorespiratory, musculoskeletal and neuromotor fitness in apparently healthy adults: guidance for prescribing exercise. Med. Sci. Sports Exerc. 43, 1334-1359. doi: 10.1249/MSS. 0b013e318213fefb

Godin, G., and Kok, G. (1996). The theory of planned behavior: a review of its applications to health-related behaviors. Am. J. Health Promot. 11, 87-98. doi: 10.4278/0890-1171-11.2.87

Gordon-Larsen, P., Nelson, M. C., and Popkin, B. M. (2004). Longitudinal physical activity and sedentary behavior trends. Am. J. Prev. Med. 27, 277-283. doi: 10. 1016/S0749-3797(04)00183-7

Hall, P. A., and Fong, G. T. (2007). Temporal self-regulation theory: a model for individual health behavior. Health Psychol. Rev. 1, 6-52. doi: 10. 1080/17437190701492437

Hillman, C. H., Erickson, K. I., and Kramer, A. F. (2008). Be smart, exercise your heart: exercise effects on brain and cognition. Nat. Rev. Neurosci. 9, 58-65. doi: 10.1038/nrn2298

Hindin, S. B., and Zelinski, E. M. (2012). Extended practice and aerobic exercise interventions benefit untrained cognitive outcomes in older adults: a meta-analysis. J. Am. Geriatr. Soc. 60, 136-141. doi: 10.1111/j.1532-5415.2011. 03761.x

Hofmann, W., Strack, F., and Deutsch, R. (2008). Free to buy? Explaining selfcontrol and impulse in consumer behavior. J. Consum. Psychol. 18, 22-26. doi: 10.1016/j.jcps.2007.10.005

Hogan, C. L., Mata, J., and Carstensen, L. L. (2013). Exercise holds immediate benefits for affect and cognition in younger and older adults. Psychol. Aging 28, 587-594. doi: 10.1037/a0032634

Hunt, A. W., Turner, G. R., Polatajko, H., Bottari, C., and Dawson, D. R. (2013). Executive function, self-regulation and attribution in acquired brain injury: a scoping review. Neuropsychol. Rehabil. 23, 914-932. doi: 10.1080/09602011. 2013.835739

Jak, A. J., Bondi, M. W., Delano-Wood, L., Wieranga, C., Corey-Bloom, J., Salmon, D. P., et al. (2009). Quantification of five neuropsychological approaches to defining mild cognitive impairment. Am. J. Geriatr. Psychiatry 17, 368-375. doi: 10.1097/JGP.0b013e31819431d5

Kramer, A. F., Hahn, S., Cohen, N. J., Banich, M. T., McAuley, E., Harrison, C. R., et al. (1999). Ageing, fitness and neurocognitive function. Nature 400, 418-419. doi: $10.1038 / 22682$

Lautenshlager, N. T., Cox, K. L., Flicker, L., Foster, J. K., van Bockxmeer, F. M., Xiao, J., et al. (2008). Effect of physical activity on cognitive function in older adults at risk for Alzheimer's disease: a randomized trial. JAMA 300, 1027-1037. doi: 10.1001/jama.300.9.1027

Liu-Ambrose, T., Nagamastu, L. S., Graf, P., Beattie, B. L., Ashe, M. C., and Handy, T. C. (2010). Resistance and training and executive functions: a 12 month randomized control trial. Arch. Intern. Med. 170, 170-178. doi: 10. 1001/archinternmed.2009.494

McAuley, E., and Blissmer, B. (2000). Self-efficacy determinants and consequences of physical activity. Exerc. Sport Sci. Rev. 28, 85-88.

McAuley, E., Mullen, S. P., Szabo, A. N., White, S. M., Wójcicki, T. R., Mailey, E. L., et al. (2011). Self-regulatory processes and exercise adherence in older adults: executive function and self-efficacy effects. Am. J. Prev. Med. 41, 284290. doi: 10.1016/j.amepre.2011.04.014

Nagamatsu, L. S., Handy, T. C., Hsu, L., Voss, M., and Liu-Ambrose, T. (2012). Resistance training promotes cognitive and functional brain plasticity in seniors with probable mild cognitive impairment. Arch. Intern. Med. 172, 666-668. doi: 10.1001/archinternmed.2012.379

Nimon, J. P., Anderson-Hanley, C., Arciero, P. J., DeMatteo, L., and Russo, M. (2009). "The theory of planned behavior and videogame-enhanced exercise for older adults: results from the cybercycle study," in Poster Presented at the 
30th Annual Meeting and Scientific Sessions of the Society of Behavioral Medicine (Montreal, Quebec).

Pennebaker, J. W. (1982). The Psychology of Physical Symptoms. New York, NY: Springer.

Ryan, R. M., Frederick, C. M., Lepes, D., Rubio, N., and Sheldon, K. M. (1997). Intrinsic motivation and exercise adherence. Int. J. Sport Psychol. 28, $335-354$.

Sallis, J. F., Grossman, R. M., Pinski, R. B., Patterson, T. L., and Nader, P. R. (1987). The development of scales to measure social support for diet and exercise behaviors. Prev. Med. 16, 825-836. doi: 10.1016/0091-7435(87)90022-3

Sallis, J. F., Pinkski, R. B., Grossman, R. M., Patterson, T. L., and Nader, P. R. (1988). The development of self-efficacy scales for health-related diet and exercise behaviors. Health Educ. Res. 3, 283-292. doi: 10.1093/her/3.3.283

Sechrist, K. R., Walker, S. N., and Pender, N. J. (1987). Development and psychometric evaluation of the exercise benefits/barriers scale. Res. Nurs. Health 10 357-365. doi: 10.1002/nur.4770100603

Smith, P. J., Blumenthal, J. A., Hoffman, B. M., Cooper, H., Strauman, T. A., WelshBohmer, K., et al. (2010). Aerobic exercise and neurocognitive performance: a meta-analytic review of randomized control trials. Psychosom. Med. 72, 239252. doi: 10.1097/PSY.0b013e3181d14633

Solberg Nes, L., Roach, A. R., and Segerstrom, S. C. (2009). Executive functions, self-regulation and chronic pain: a review. Ann. Behav. Med. 37, 173-183. doi: 10.1007/s12160-009-9096-5

Steffens, D. C., Fisher, G. G., Langa, K. M., Potter, G. G., and Plassman, B. L. (2009). Prevalence of depression among older Americans: the aging, demographics and memory study. Int. Psychogeriatr. 21, 879-888. doi: 10.1017/s10416102099 90044

Strauss, E., Sherman, E. M. S., and Spreen, O. (2006). A Compendium of Neuropsychological Tests: Administration, Norms and commentary. 3rd Edn. NY: Oxford University Press.
Stroop, J. R. (1935). Studies of interference in serial verbal reactions. J. Exp. Psychol. 18, 643-662. doi: 10.1037/h0054651

Trujillo, K. M., Brougham, R. R., and Walsh, D. A. (2004). Age differences in reasons for exercise. Curr. Psychol. 22, 348-367. doi: 10.1007/s12144-004-1040-z

Van der Elst, W., Van Boxtel, M. P., Van Breukelen, G. J., and Jolles, J. (2006). The stroop color-word test influence of age, sex and education; and normative data for a large sample across the adult age range. Assessment 13, 62-79. doi: 10. $1177 / 1073191105283427$

Withall, J., Jago, R., and Fox, K. R. (2011). Why some do but most don't. Barriers and enablers to engaging low-income groups in physical activity programmes: a mixed methods study. BMC Public Health 11:507. doi: 10.1186/1471-2458-11507

Conflict of Interest Statement: The authors declare that the research was conducted in the absence of any commercial or financial relationships that could be construed as a potential conflict of interest.

Received: 01 July 2014; accepted: 19 November 2014; published online: 05 December 2014.

Citation: Anderson-Hanley C, Arciero PJ, Barcelos N, Nimon J, Rocha T, Thurin M and Maloney M (2014) Executive function and self-regulated exergaming adherence among older adults. Front. Hum. Neurosci. 8:989. doi: 10.3389/fnhum.2014.00989 This article was submitted to the journal Frontiers in Human Neuroscience.

Copyright $\odot 2014$ Anderson-Hanley, Arciero, Barcelos, Nimon, Rocha, Thurin and Maloney. This is an open-access article distributed under the terms of the Creative Commons Attribution License (CC BY). The use, distribution and reproduction in other forums is permitted, provided the original author(s) or licensor are credited and that the original publication in this journal is cited, in accordance with accepted academic practice. No use, distribution or reproduction is permitted which does not comply with these terms. 